\title{
La sauvegarde et la valorisation du patrimoine culturel au Cameroun
}

The safeguarding and the development of Cameroon's Cultural Heritage

Estelle Piou, Sylvain Djache Nzefa, Flaubert Ambroise Taboue Nouaye et Anita Kamga Fotso

\section{(2) OpenEdition}

\section{Journals}

Édition électronique

URL : http://journals.openedition.org/ocim/1026

DOI : $10.4000 /$ ocim. 1026

ISSN : 2108-646X

Éditeur

OCIM

Édition imprimée

Date de publication : 1 janvier 2012

Pagination : $30-39$

ISSN : 0994-1908

Référence électronique

Estelle Piou, Sylvain Djache Nzefa, Flaubert Ambroise Taboue Nouaye et Anita Kamga Fotso, « La sauvegarde et la valorisation du patrimoine culturel au Cameroun », La Lettre de I'OCIM [En ligne], 139 | 2012, mis en ligne le 01 janvier 2014, consulté le 01 mai 2019. URL : http:// journals.openedition.org/ocim/1026; DOI : 10.4000/ocim.1026 


\title{
La sauvegarde et la valorisation du patrimoine culturel au Cameroun
}

\author{
Estelle Piou, Sylvain Djache Nzefa,
} Flaubert Ambroise Taboue Nouaye et Anita Kamga Fotso *

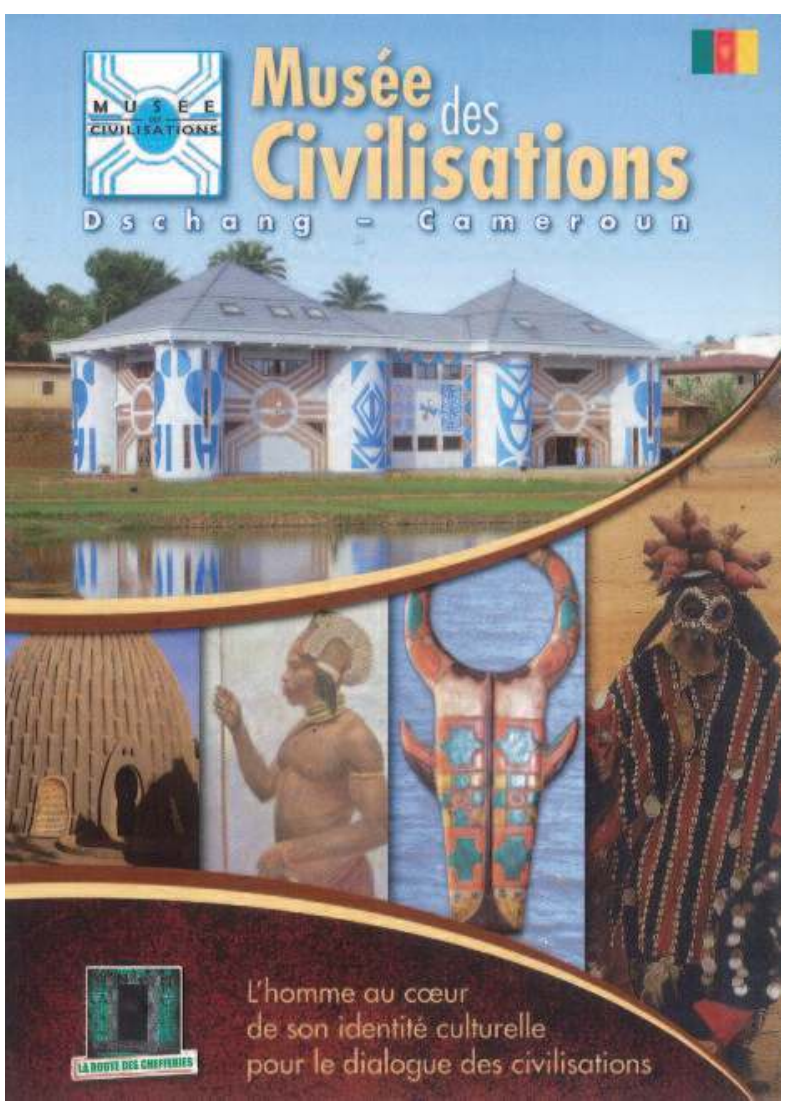

Le dépliant de présentation de "La Route des Chefferies » ○ PRDC

* Estelle Piou est directrice du Patrimoine de « La Route des Chefferies » Sylvain Djache Nzefa est architecte-urbaniste-scénographe, fondateur principal et coordonateur général de « La Route des Chefferies» Flaubert Ambroise Taboue Nouaye est conservateur et directeur du musée des Civilisations Anita Kamga Fotso est responsable marketing et communication de « La Route des Chefferies »
Le programme "La Route des Chefferies » a pour objectif de créer un pôle culturel et touristique inédit au Cameroun afin d'amener les populations à se réapproprier leur patrimoine tout en contribuant à leur développement économique et social : cette expérience originale en Afrique, relatée ici, constitue un formidable terrain d'étude où les problématiques muséales et plus largement patrimoniales sont interrogées dans un contexte particulier.

\section{Un contexte particulier}

Le Cameroun, pays d'Afrique Centrale, est communément présenté comme l'Afrique en miniature et très récemment par le slogan "Toute l'Afrique dans un seul pays ». Il doit cet attribut à la variété de ses végétations et paysages du Nord au Sud, de l'Est à l'Ouest, et à la diversité ethnique - plus de 200 ethnies - de sa population entre autres. Les populations sont organisées en royaumes, clans, villages, lamidats... généralement dirigés par un chef, un sultan ou un lamido. Comme beaucoup de pays africains, les sociétés traditionnelles du Cameroun ont connu des sorts multiples liés aux événements de l'Histoire lors de la traite négrière, de la colonisation et de la marche vers l'indépendance. Par ailleurs, avec le développement des échanges mondiaux, le Cameroun a fait l'objet de nombreuses études d'explorateurs, ethnologues, médecins, missionnaires, industriels, commerçants... et a ainsi subi, sur le plan patrimonial, les pillages, les extorsions, les réquisitions, les trocs, les 
achats ou même les dons d'objets en remerciements de services rendus. À ceci s'ajoute le manque de moyens pour l'entretien et la réhabilitation du patrimoine bâti dans les chefferies. Quelles qu'en soient les raisons, le constat est le même à savoir que la perte du patrimoine matériel a contribué à la perte des valeurs identitaires et séculaires des sociétés composant le Cameroun.

Compte tenu de tous ces paramètres, au lendemain de l'indépendance, le jeune État du Cameroun n'a pas jugé nécessaire d'inscrire dans ses priorités la protection du patrimoine. Un ministère de la Culture est pourtant créé avec plusieurs directions dont une spécifique au patrimoine. Mais aucune action concrète n'est menée en faveur de la protection du patrimoine. Même aujourd'hui, après 50 ans d'indépendance, l'État, censé être le moteur déclencheur de la préservation et de la valorisation de ses richesses, ne joue pas complètement son rôle. Les lois spécifiques à la régulation du patrimoine culturel, artistique et touristique en général sont désuètes, sinon inexistantes. Le Cameroun souffre d'une véritable absence de politique culturelle et patrimoniale.

Alors que le pays a ratifié depuis 1982 la convention du patrimoine mondial de l'UNESCO pour la protection des biens culturels et naturels, puis en avril 2008 la convention de 2003 de l'UNESCO sur la sauvegarde du patrimoine culturel immatériel, on observe une absence de dispositions spécifiques définissant les types de patrimoines culturels à conserver. Le premier inventaire général du patrimoine camerounais a été initié par le gouvernement en 2001 c'est-à-dire plus de 40 ans après son accession à la souveraineté internationale. Appuyé par la France, il démarre dans les régions des Grassland (Ouest Cameroun) et Fang-Béti (Sud Cameroun) et s'interrompt brusquement en 2003. Cette action a été très peu fructueuse. L'inventaire a ensuite été repris dans l'aire culturelle soudano-sahélienne après la formation de 156 jeunes enquêteurs en 2006, mais l'opération s'est soldée par un échec.

Malgré ce contexte politique difficile, la vitalité des expressions artistiques et culturelles survit. "Avec des bouts de ficelles et une passion à toute éprewve, des artistes et opérateurs culturels inventent des solutions pour surmonter les obstacles " ${ }^{(1)}$. On assiste ainsi à un développement des activités culturelles souvent dans l'informel, ou avec l'appui de la diaspora, des ONG internationales ou des organismes de coopération. « La Route des Chefferies » va se frayer un chemin de cette manière. D’abord lancée par la diaspora de Nantes, elle retient l'attention de partenaires français, de la communauté internationale, des entreprises et des élites locales contribuant efficacement à son déploiement.

\section{Les chefferies au cœur de la démarche}

Malgré les affres du temps, les chefferies ont su résister aux oppressions diverses. En 1960, le gouvernement camerounais, nouvellement créé, reconnaît les chefferies comme des auxiliaires administratifs servant de lien entre l'administration et les populations du village où elles ont encore autorité pour rendre la justice traditionnelle.

Les chefferies traditionnelles au Cameroun sont des entités politiques, sociales et culturelles, caractérisées par un territoire bien délimité, un peuple avec son histoire, ses us et coutumes. Elles sont placées sous la haute autorité d'un chef. Dans l'aire culturelle des Grassfield principalement (régions administratives de l'Ouest et du Nord-Ouest), les chefs Bamiléké (appelés roi, fo, fon ou sultan... selon les lieux) sont des personnages très respectés et craints de leur population. Ce sont les principaux dépositaires du patrimoine, qu'il soit matériel ou immatériel, parce qu'ils constituent le lien entre le monde des ancêtres et le monde des vivants (composé des humains et des animaux). Dans les Grassfield, les peuples pratiquent avant tout le culte des ancêtres. En termes de territoire, l'espace de vie du chef est le point focal où la vie culturelle foisonne.

Le patrimoine culturel au Cameroun est encore aujourd'hui constitué à $85 \%$, de ce que les ethnologues appellent "une collection dont le but est de

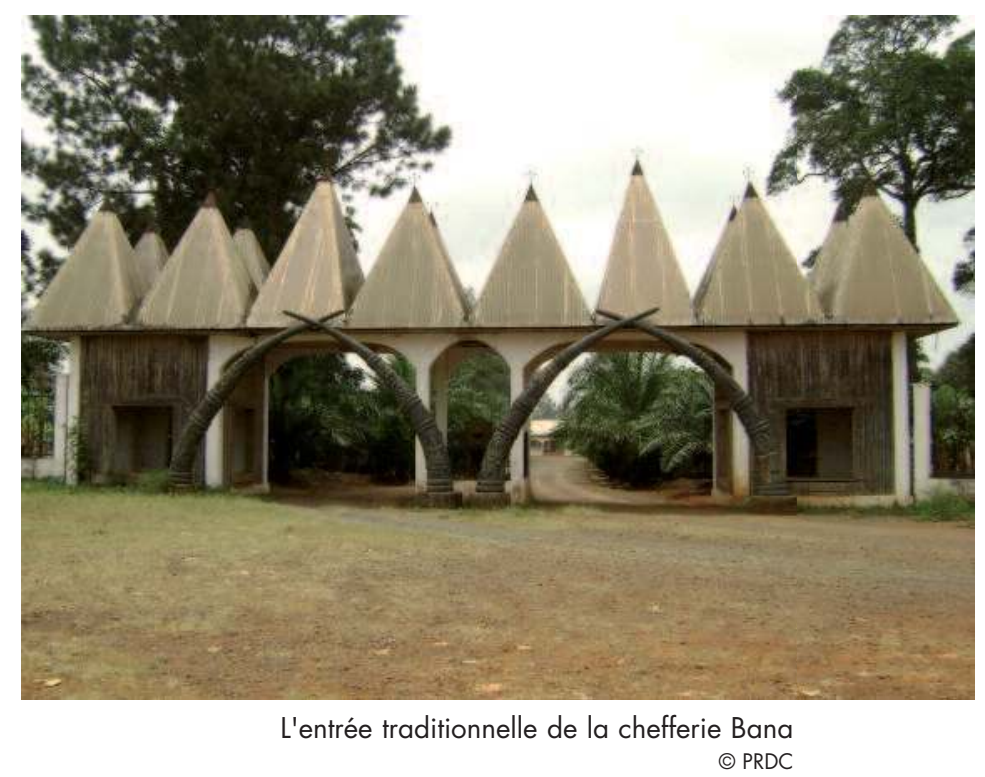

(c) PRDC 
faire connaître et comprendre la société, la nature, l'histoire et la culture d'une unité de population définie par une identité linguistique et anthropologique exprimée dans une communauté de traits matériels et spirituels » (2). Autrement dit, cette culture vivante continue de produire les supports entretenant sa vitalité. Les œuvres créées sont pour l'essentiel le reflet de cette vie structurée de manière séculaire (organisation sociale, religieuse, us et coutumes...), mettant ainsi les artistes au service de la société qui ellemême se réapproprie l'objet créé pour le transformer en patrimoine communautaire.

Comme toute culture vivante, la culture Bamiléké évolue indéniablement avec les changements de société. Pour ne pas voir leurs traditions s'éteindre, tout en s'adaptant à la modernisation, les chefs se trouvent alors confrontés à plusieurs paradoxes et doivent surmonter plusieurs difficultés. D’une part, ils constatent une déconsidération pour les valeurs culturelles locales et un déracinement de la population principalement dus à l'exode rural. La culture est de moins en moins transmise aux nouvelles générations qui préférent les biens de consommation modernes. D'autre part, il existe toujours une forme de pillage (qui ne porte pas son nom) du patrimoine matériel. Par ailleurs, les chefferies traversent une crise caractérisée par une gestion désordonnée et souffrent d'une faiblesse institutionnelle, matérielle, financière et organisationnelle. Le croisement entre autorité traditionnelle et autorité administrative constitue parfois une source de dysfonctionnement. Tous ces aspects sont développés dans l'ouvrage Les chefferies Bamiléké dans l'enfer du modernisme. Une chefferie de demain, écrit par Sylvain Djache Nzefa, architecte camerounais, qui dans une démarche d'ethnologue-sociologue sonne l'alarme en 1994 avec son diagnostic présentant les chefferies prises dans l'engrenage du « modernisme ». Des structures "en décadence en ce qui concerne les aspects sociopolitiques, religieux, économiques... Elles traversent depuis longtemps, ainsi que toute la société camerounaise... une violente crise d'identité : il s'agit d'un véritable chaos culturel » (3). Ce constat alarmant constitue alors le détonateur d'une réflexion qui avait pour but principal de donner aux chefferies leurs lettres de noblesse sur tous les aspects de leurs patrimoines (matériel et immatériel).

\section{Un concept créé sur mesure}

Le diagnostic posé par Sylvain Djache Nzefa est au cœur de la charte de « La Route des Chefferies » élaborée en 2006. Il en ressort un besoin urgent d'inventorier

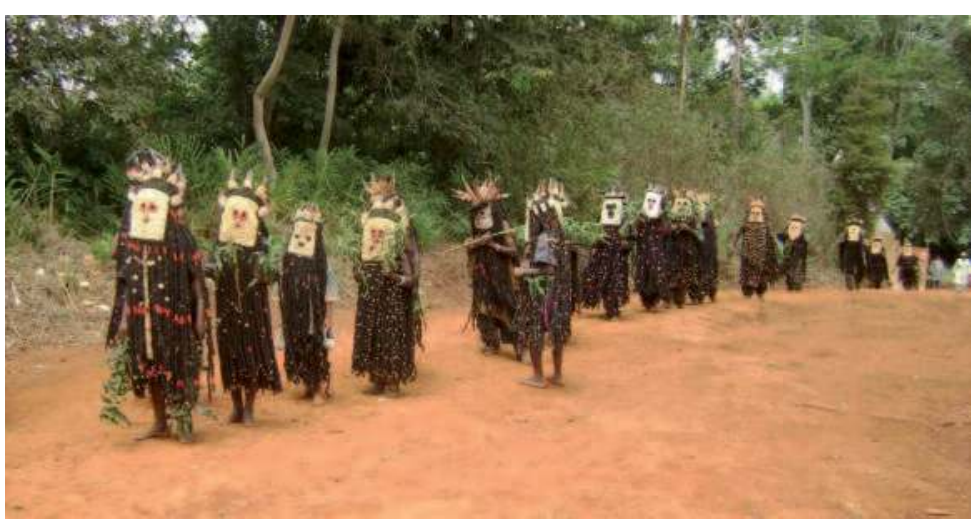

Une sortie de la société secrète Kun'gang lors du festival «Chepan » de la chefferie Bamendjou en avril 2009

() PRDC

le patrimoine, de sensibiliser et d'informer les populations locales et les diasporas sur leurs origines culturelles, de montrer que la protection du patrimoine n'est en rien un frein à la modernisation, de structurer les chefferies en matière de gestion et d'organisation, et de développer les échanges et partenariats.

C'est dans cette logique que s'inscrit et s'écrit le Programme de «La Route des Chefferies » (PRDC). Dynamique culturelle, touristique et économique, la volonté est de réunir une grande variété de protagonistes avec comme actrices principales les chefferies traditionnelles. L'originalité du PRDC est de placer l'homme au cœur de son identité culturelle dans un esprit d'ouverture et de dialogue interculturel. Il propose ainsi des espaces d'interprétation proposant une lecture : - des fondements de la cosmogonie et de la cosmologie des chefferies Bamiléké ;

- de l'art et de l'architecture des chefferies dans l'espace et le temps ;

- des sociétés traditionnelles du Cameroun et d'ailleurs ;

- de l'homme dans son environnement naturel.

Le PRDC crée ainsi les outils nécessaires à la préservation, la conservation, la restauration, la promotion du patrimoine matériel et du patrimoine immatériel. Il mise également sur la valorisation du patrimoine et l'aménagement culturel du territoire pour développer la région et la promouvoir sur le plan touristique. Les objectifs du PRDC sont multiples :

- sensibiliser la population camerounaise à ses valeurs culturelles afin d'aboutir à une réappropriation de son patrimoine matériel et immatériel ;

- innover par la création d'un pôle culturel inédit en Afrique noire ;

- mettre en place un programme de sécurisation et d'inventaire du patrimoine ; 
- organiser un réseau pour une démarche concertée sur le territoire ;

- répondre aux attentes de développement économique rural et urbain des populations afin d'améliorer leurs conditions sociales (principalement par la création d'emplois).

« La Route des Chefferies » repose sur une charte fondatrice plaçant les chefs traditionnels au cœur de la démarche. Par leur signature, ils marquent leur engagement moral, culturel, identitaire à respecter le concept, les objectifs et les principes généraux du programme. Signée en octobre 2006 par 25 chefs, la charte « La Route des Chefferies » regroupe aujourd'hui plus d'une quarantaine de chefferies sur 106.

Après plusieurs années de travail, le PRDC rassemble un ensemble d'actions, de structures et d'outils. Tous les équipements réalisés s'adressent en premier lieu aux Camerounais, les jeunes, les familles, les étudiants, chercheurs, universitaires et la diaspora revenant au village durant les congés scolaires.

Bien entendu, les touristes constituent également une cible principale. Bien que le Cameroun ne soit pas un pays où le tourisme international soit encore très développé, il bénéficie en revanche d'un important tourisme d'affaire et d'un tourisme d'expatriés principalement basés à Yaoundé (capitale administrative) et Douala (capitale économique). Nous verrons plus loin que l'étude de fréquentation réalisée à un an d'ouverture du musée des Civilisations montre que les objectifs fixés en termes de publics sont en passe d'être remplis.

\section{Le musée des Civilisations, au cœur du Programme "La Route des Chefferies"}

Le musée des Civilisations est un centre d'interprétation des civilisations du Cameroun ouvert au public depuis le

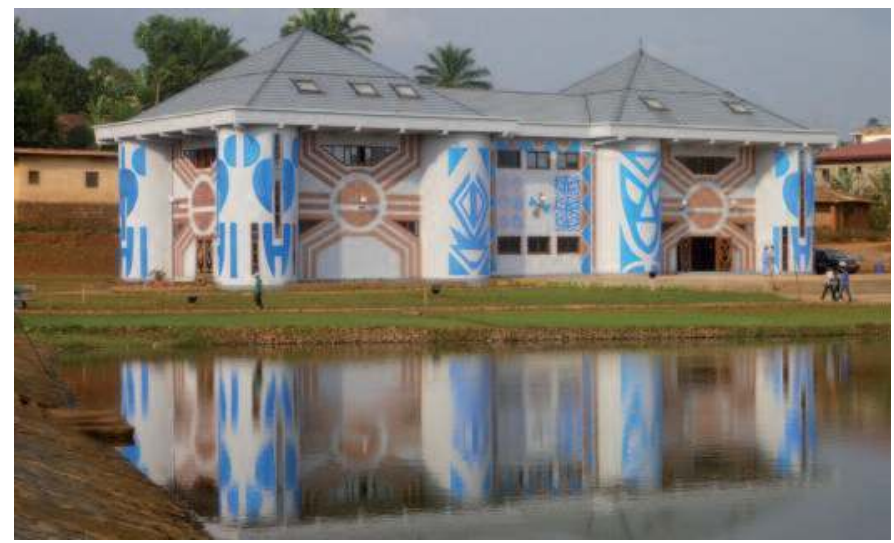

L'entrée du musée des Civilisations (c) PRDC
20 novembre 2010. Édifice symboliquement fort dans une région verdoyante aux patrimoines naturel et culturel particulièrement riches, le musée des Civilisations est implanté sur les bords du lac de Dschang devenu un lieu authentique de loisirs et de culture avec sa base nautique (2005), son terrain de beach volley et son aire de jeu (2006), son centre artisanal (2010) et prochainement un Jardin des Civilisations. Le musée des Civilisations (MDC) est une grande réalisation communautaire à vocation régionale, nationale et universelle. Il constitue l'épicentre du concept de «La Route des Chefferies».

Après une présentation introductive du Cameroun au fil des siècles, le visiteur est invité à découvrir le peuple camerounais dans son identité à la fois plurielle et fusionnelle : les peuples de la forêt avec leur expression totémique, les peuples de la mer avec la renaissance du Ngondo - festival culturel et cultuel -, les peuples soudano-sahéliens avec leurs lamidats, chefferies, sultanats et les peuples des Grassfield avec leurs chefferies mystérieuses. Il se veut un centre d'interprétation favorisant une approche intégrée du patrimoine culturel permettant aux visiteurs, surtout les communautés locales, de renouer avec leurs racines tout en abordant les cultures des autres régions. Cette approche participe d'une tolérance culturelle, vecteur important de la diversité des cultures. Le lieu offre aux visiteurs un espace de $1200 \mathrm{~m}^{2}$ se répartissant entre expositions permanente et temporaire, ateliers pédagogiques, boutiques, salle de conférences et bientôt un centre de documentation.

\section{Une architecture forte}

L'architecture africaine contemporaine, signée par l'architecte Sylvain Djache Nzefa, principal fondateur de "La Route des Chefferies », est caractérisée par la symphonie des symboles : araignée, buffle, éléphant, écritures.

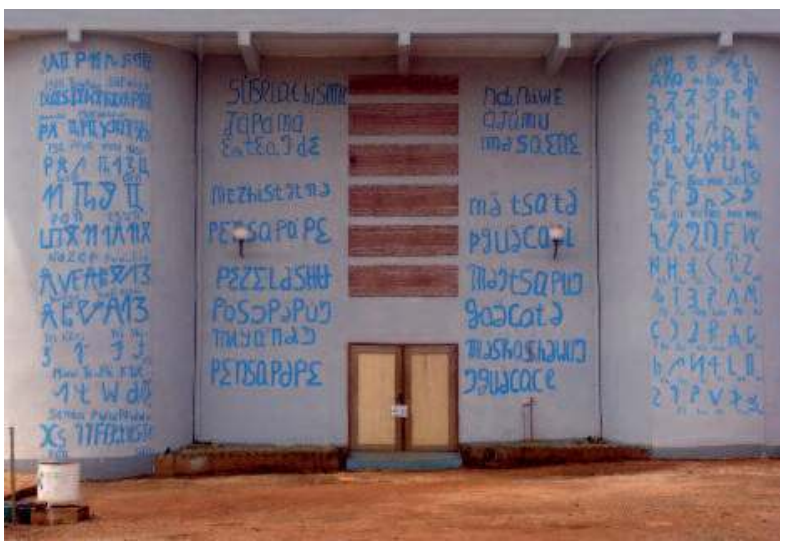

La façade Est du musée, avec les écritures en plusieurs langues locales du Cameroun () PRDC 
Le musée des Civilisations, de par ses fonctions multiples, est détenteur d'histoire, de savoir, d'intelligence, de connaissance. Dans le monde des vivants, dans la cosmogonie Bamiléké, les hommes et les animaux dialoguent, et ce dialogue va se faire aussi dans la représentation. Chez les vivants, celui qui possède la somme de toutes les expériences est le vieux. Partout en Afrique noire, le vieux «était» considéré comme le sage, le connaisseur, celui qui « détenait » la vérité, par ses expériences multiples. C'est sa connaissance qui est symbolisée à travers l'araignée. Selon Pierre Harter : "L'araignée-mygale, symbole de la connaissance, prend souvent la forme d'un petit cercle simple ou double, doté de quatre pattes croisées et non huit, ce qui permet de composer sur certaines coiffures de masques ou certaines gravures de cornes à libation, des sortes de filets à mailles losangiques ou carrées ». L'araignée apparaît donc comme l'élément central de l'architecture du musée des Civilisations : elle est ici prise comme un élément architectonique qui rythme la façade.

La façade est traitée comme un masque à travers des moulures de couleur bleu. Il y a une volonté de masquer ce qui se trouve derrière. N'oublions pas que chez le négro-africain, les masques parlent ; le porteur est possédé par son masque. Ici, le porteur, c’est le musée. Le masque représentant l'araignée exprime le contenu de ce bâtiment, c'est-à-dire la connaissance. Les masques de buffles et d'éléphants symbolisent la force, la puissance, la grandeur et la richesse. Une autre façade du musée est recouverte d'alphabets et du mot «bienvenue » écrit dans plusieurs langues et dialectes parlés au Cameroun.

Lacte de bâtir ou de conceptualiser objets et habitat est considéré comme support de savoir et de compréhension de l'autre. Il amène à revoir tout ce qui se joue dans la constitution et les fondements des différentes civilisations du Cameroun. Le visiteur est ainsi amené à s'interroger sur son identité culturelle par une meilleure connaissance de soi, facilitant ainsi le dialogue entre les civilisations.

\section{Une muséologie africaine expérimentée}

Le musée des Civilisations développe une expérience muséographique africaine visant à mettre le public au cœur de la démarche à travers des supports didactiques et des décors contextuels permettant ainsi de s'immerger au cœur des cultures camerounaises. Il ne s'agit pas tant de donner à voir mais bien de créer une expérience de visite en provoquant sensations et émotions.

Réalisé par une équipe franco-camerounaise, le contenu de l'exposition s'appuie sur une démarche scientifique

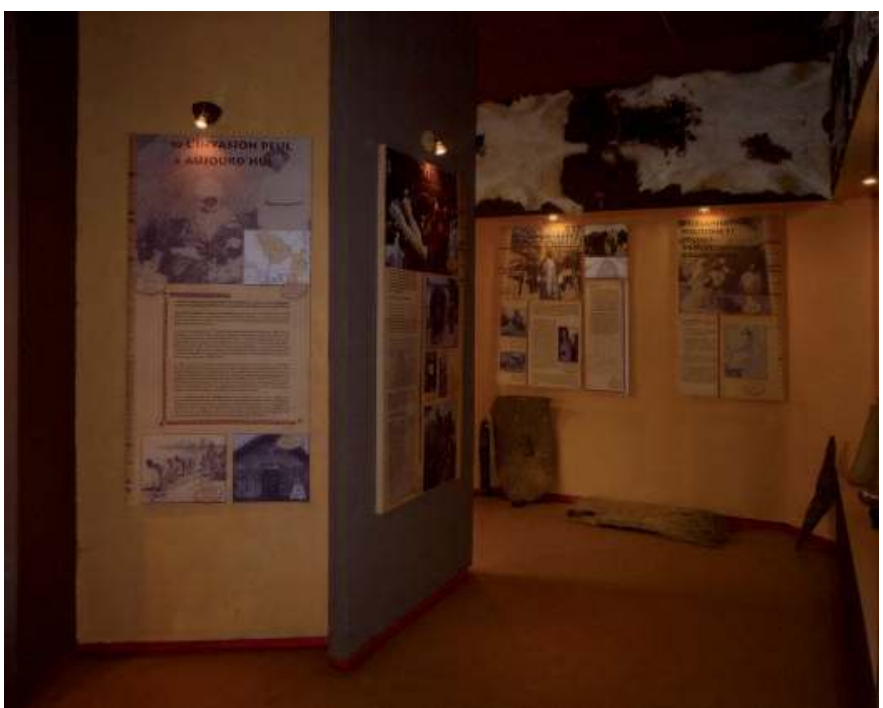

L'organisation des sociétés du Nord Cameroun dans l'espace consacré aux peuples soudano-sahélien au musée des Civilisations. (c) PRDC

et interdisciplinaire (histoire, archéologie, ethnographie, sociologie, architecture et patrimoine...). Dynamique et pédagogique, l'exposition a été conçue, dans l'espace et dans le temps, de manière à pouvoir intégrer les événements d'actualité. Avec plus de 500 objets, 1500 illustrations, des espaces vidéo, la muséographie intègre de manière importante les matériaux africains traditionnels travaillés dans une approche contemporaine par les artisans locaux (bambou vernis ou brûlé, écorce de bananier, écorce d'obom, cauris, perles, raphia, terre, noix de coco...).

Le musée des Civilisations ne trouve pas sa justification dans l'existence d'une collection d'objets qui lui serait propre. Les pièces présentées viennent illustrer le discours choisi par les concepteurs et proviennent pour l'essentiel des prêts et dépôts des chefferies. Elles sont également constituées de copies d'objets emblématiques d'une aire culturelle, des commandes spécifiques passées auprès des artistes du terroir, voire des acquisitions d'objets contemporains. Chacun de ces objets a été choisi pour sa qualité pédagogique, son authenticité et sa valeur culturelle.

\section{Les autres activités de sauvegarde et de valorisation du patrimoine}

\section{Les inventaires}

Des inventaires du patrimoine culturel dans quatorze chefferies ont été réalisés entre 2007 et 2010. Une troisième phase doit démarrer début 2012. Cette activité bénéficie d'une forte mobilisation des enseignants 
et des étudiants de l'université de Dschang pour les travaux sur le terrain. Il s'agit ici de l'inventaire du patrimoine matériel (objets, architecture) et immatériel (danses et rites) de ces chefferies. Une monographie répertoriant l'histoire de la chefferie, les lieux sacrés et les sites touristiques complète ce travail.

\section{Les cases patrimoniales}

Après les inventaires arrive la phase de conservation et de diffusion du patrimoine. Pour cela, le PRDC a entrepris la mise en place ou le réaménagement des musées communautaires au sein des chefferies labellisés « cases patrimoniales ». Ces musées sont établis à la demande du chef marquée par sa forte implication et celle de sa communauté. En effet, le bâtiment est à la charge de la chefferie. Une fois le projet défini en concertation avec le PRDC qui peut apporter une expertise en architecture et suivi des travaux, les financements sont ensuite recherchés pour la construction ou la réhabilitation d'un bâtiment existant. Les chefs mobilisent ainsi différents partenaires dont les élites de leur communauté réunies en comité de développement ; elles contribuent ainsi à la prise en charge de certains postes de dépenses liés à la mise en œuvre de la case patrimoniale.

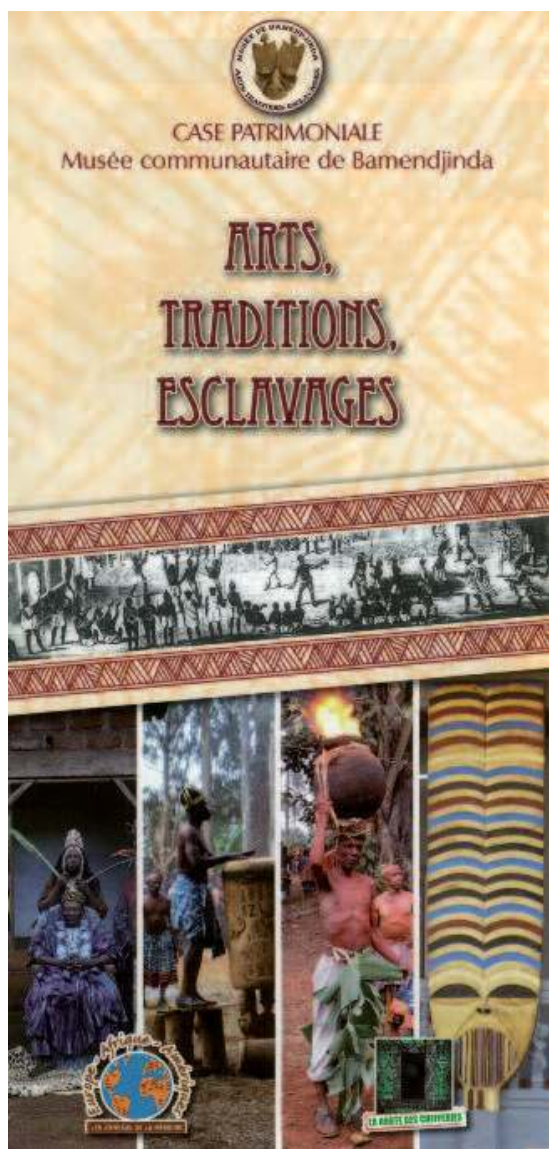

Le dépliant thématique de la case patrimoniale de Bamendjinda
Chaque case patrimoniale développe une thématique qui lui est propre en relation avec l'histoire ou l'originalité de la chefferie ou simplement un thème cher au chef. C'est ainsi que les sujets tels que le phénomène des esclavages (coutumier et négrier), le rôle de la forge, l'art de la chasse et les arts traditionnels et contemporains ont été traités. De nouvelles thématiques comme l'homme et ses rapports avec la nature et son environnement sont à l'étude. Chaque thème est défini par le chef qui doit par conséquent constituer un comité scientifique spécialisé sur la thématique développée, et identifier le personnel qui travaillera au quotidien dans son musée. Le PRDC, en dehors de son apport scientifique complémentaire aux travaux réunis par la chefferie, apporte son expertise en termes de conception et réalisation muséographiques ainsi que dans le domaine de la formation du personnel. Il soutient également le fonctionnement du musée pendant une période déterminée. Quatre cases patrimoniales sont ainsi ouvertes au public dans les chefferies de Bamendjinda, Bandjoun, Bamendjou et Bangoua.

\section{Les formations professionnelles}

Dans le but de professionnaliser le fonctionnement de toutes ces nouvelles structures, le PRDC a initié une série de formations des acteurs locaux dont les chefs traditionnels, dans les domaines de la gestion de musée, l'accueil des publics, la conservation préventive et la médiation culturelle. Cette mise à niveau des compétences des acteurs locaux contribue efficacement à leur implication dans la cause d'une valorisation « équitable » de leur patrimoine. Les quatre sessions de formation, d'une semaine en moyenne, organisées en 2009 et 2010, ont toutes bénéficié de l'expertise de l'École du Patrimoine Africain de Porto-Novo au Bénin qui a dépêché chaque fois une équipe de spécialistes sur les questions abordées.

\section{La médiation culturelle}

L'équipe du PRDC développe, depuis 2008 avant l'ouverture même du musée des Civilisations, un ensemble d'activités de médiation culturelle orientées vers le public scolaire. Ces actions visent à développer le binôme "musée-école » et à accompagner le système pédagogique à stimuler de nouveaux types d'apprentissages. Depuis l'ouverture du musée des Civilisations, de nouvelles activités sont mises en place pour les scolaires et les étudiants.

Par ailleurs, le PRDC a mis en place des expositions temporaires et organisé des conférences en partenariat avec sa voisine l'Alliance franco-camerounaise 


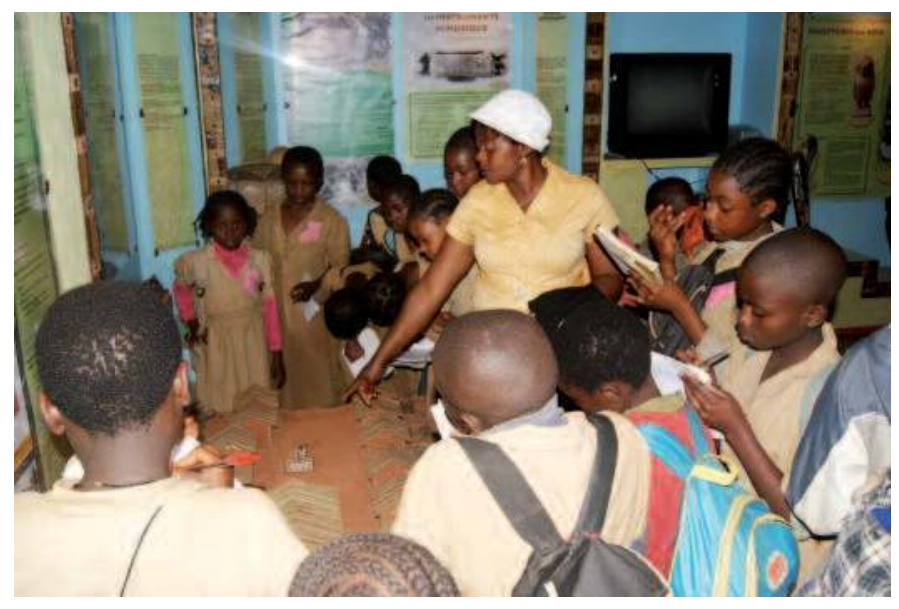

Une séance pédagogique avec une classe de collège au musée des Civilisations (c) PRDC

de Dschang. Il vient également en appui des cases patrimoniales qui développent chacune un programme d'activités pédagogiques et culturelles (parcours jeune dans les expositions, ateliers de vannerie, de perlage des objets ou de tressage du bambou...).

\section{Et pour la pérennité...}

Dans le but d'assurer la pérennité des équipements du PRDC et à long terme son autonomie financière, de valoriser les savoir-faire des communautés et participer à l'amélioration de leur bien-être, le PRDC a développé un modèle économique composé d'un ensemble d'outils. Ceux-ci sont investis au sein du circuit « La Route des Chefferies », qui devient ainsi un produit touristique. Dans ce volet, un centre artisanal a été

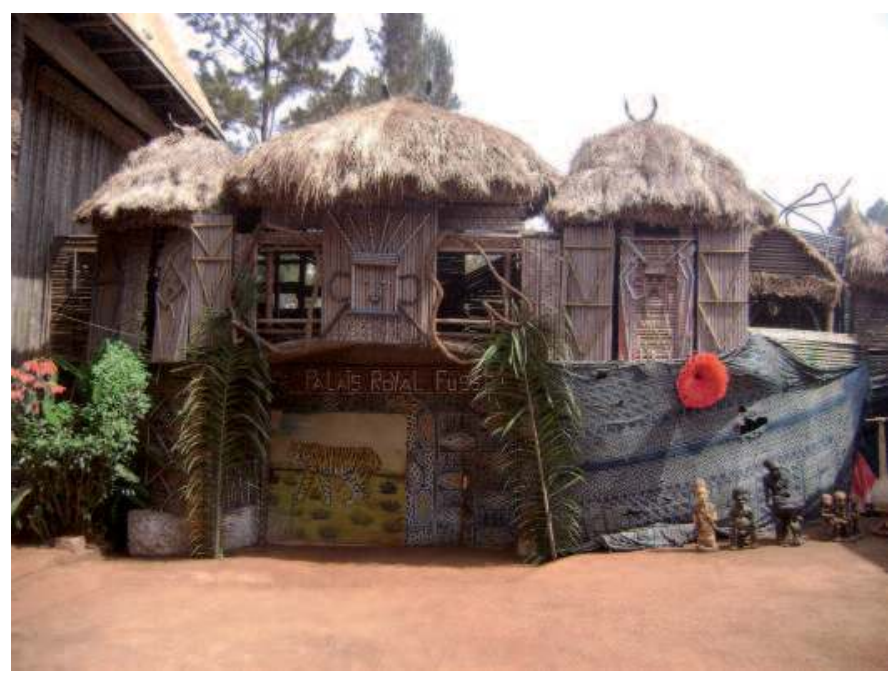

La décoration de la cour du palais royal de la chefferie Bafoussam (c) PRDC construit sur le site du musée des Civilisations. Il constitue un espace d'exposition pour la valorisation des savoir-faire artisanaux camerounais. Réparties en 8 boukarous de $16 \mathrm{~m}^{2}$, ces cases sont organisées par corps de métiers artisanaux (vannerie, poterie, sculpture sur bois, textile, agro-alimentaire...) en plus de la boutique du musée qui propose des produits dérivés du musée des Civilisations et de « La Route des Chefferies » en général. Le centre artisanal possède également une cour centrale dallée, de $112 \mathrm{~m}^{2}$, pouvant accueillir des foires et expositions temporaires. Dans la phase 2, un bâtiment central viendra compléter cette réalisation. Au sein des cases patrimoniales, des espaces de vente sont également prévus.

Toujours à l'initiative des chefs, des cases d'hôtes, logements originaux, sont initiées au sein des chefferies. C'est une forme d'hébergement qui permet de capitaliser le tourisme solidaire de plus en plus pratiqué par les touristes et valorisé par « La Route des Chefferies». Ce dispositif, en plus de créer une nouvelle source de revenus pour les chefferies, favorise la proximité avec les populations et la découverte culturelle.

Au-delà de tous ces espaces, des outils ont été mis sur pied pour vendre la destination Ouest du Cameroun à travers le circuit « La Route des Chefferies ». Il s'agit notamment d'un plan-guide culturel, réédité à 10000 exemplaires, qui comprend un fond de carte routière de l'Ouest, une présentation globale des us et coutumes caractéristiques des Grassfield, des zooms sur certaines chefferies traditionnelles, des principaux sites naturels d'intérêt touristique, des photographies de quelques rois, de trônes, de sièges royaux et d'artefacts culturels. C'est un outil qui autant que les supports de communication (dépliants réalisés à 5000 exemplaires pour chaque case patrimoniale et 10000 exemplaires pour le musée des Civilisations), contribue à communiquer sur «La Route des Chefferies » audelà de la région de l'Ouest et devient un dispositif indispensable pour les voyageurs, les étudiants, les professionnels du patrimoine et les Camerounais d'une manière générale. Le PRDC est également doté d'un site Internet, plateforme incontournable pour une meilleure valorisation de ses activités et réalisations : www.routedeschefferies.com.

\section{Du dialogue Nord/Sud à la coopération Sud/Sud}

À travers ses années d'expérience et les obstacles surmontés, le PRDC a su mettre en place une méthodologie adaptée reposant sur plusieurs principes que sont : 
Rattaché au musée des Civilisations, le pôle de conservation intervient auprès de toutes les cases patrimoniales de « La Route des Chefferies ». Les moyens ne permettent pas aujourd'hui de mettre en place une réelle politique de conservation préventive et encore moins de restauration. Cependant, un minimum de procédures est défini afin que de bonnes pratiques puissent être respectées au quotidien. Notons par exemple :

- le traitement des objets en bois présentés dans les expositions et en réserves avec une fiche de suivi pour chaque objet ;

- l'aménagement de salles de réserves en tentant de réunir un minimum de conditions favorables : obturation des fenêtres lorsque celles-ci n'ont que des grilles par exemple, colmatage des fuites d'eau, installation d'étagères pour éviter la pose d’objets au sol, dépoussiérage régulier... ;

- la mise à jour des inventaires et la poursuite de la recherche documentaire ;

- le suivi et l'accompagnement régulier des équipes dans leur travail.

La directrice adjointe du musée des Civilisations en charge de la conservation vient d'effectuer un stage au musée du Quai Branly où elle a pu étudier la problématique de la conservation préventive des objets au Cameroun et l'archivage des documents. Une stratégie va être élaborée et permettra, nous l'espérons, de lever les fonds pour passer à la phase concrète.

Un centre de documentation est également à l'étude et devrait être aménagé dans le courant de l'année 2012 .
- la concertation entre les acteurs pour renforcer le dialogue et la pertinence de l'intervention du programme visant à enclencher une dynamique durable de maîtrise locale du développement ;

- le renforcement des capacités et la structuration des acteurs locaux à travers les formations et accompagnements personnalisés ;

- l'accompagnement des logiques Sud/Sud et Sud/ Nord à travers le partage d'expériences entre les acteurs sur les problématiques développées ;

- la capitalisation des informations, des relevés et de la connaissance scientifique culturelle. Le PRDC vise à jouer un rôle déterminant dans le développement de nouvelles approches et de plaidoyers de la culture camerounaise en particulier et africaine en général.

Un comité de pilotage se tient une fois par an et réunit les chefs signataires de la charte, les structures porteuses du PRDC et des intervenants extérieurs tels que les préfets et municipalités où sont implantées les structures du PRDC et les administrations déconcentrées de l'État (Culture, Tourisme et Artisanat).

Une autre particularité du PRDC réside dans ses ressources humaines. L'équipe constituée est pluridisciplinaire et internationale et réunit des expertises variées en conservation, architecture, médiation culturelle, valorisation du patrimoine, management de la culture, communication, mécénat, tourisme culturel, aménagement du territoire, sans compter les chercheurs et scientifiques issus des sciences humaines (histoire, géographie, archéologie, sociologie, ethnologie, anthropologie). Le PRDC compte ainsi une centaine de personnes avec une grande représentativité des femmes aux postes à responsabilité, soit $54 \%$ du nombre total de responsables.

Sur le plan des réseaux culturels et scientifiques, des partenariats sont également développés. Des coopérations et échanges s'établissent ainsi avec l'université de Dschang, la formation Sciences du Patrimoine de l'Institut supérieur du Sahel de l'université de Maroua au Cameroun, l'École du Patrimoine Africain au Bénin et le musée du Quai Branly en France.

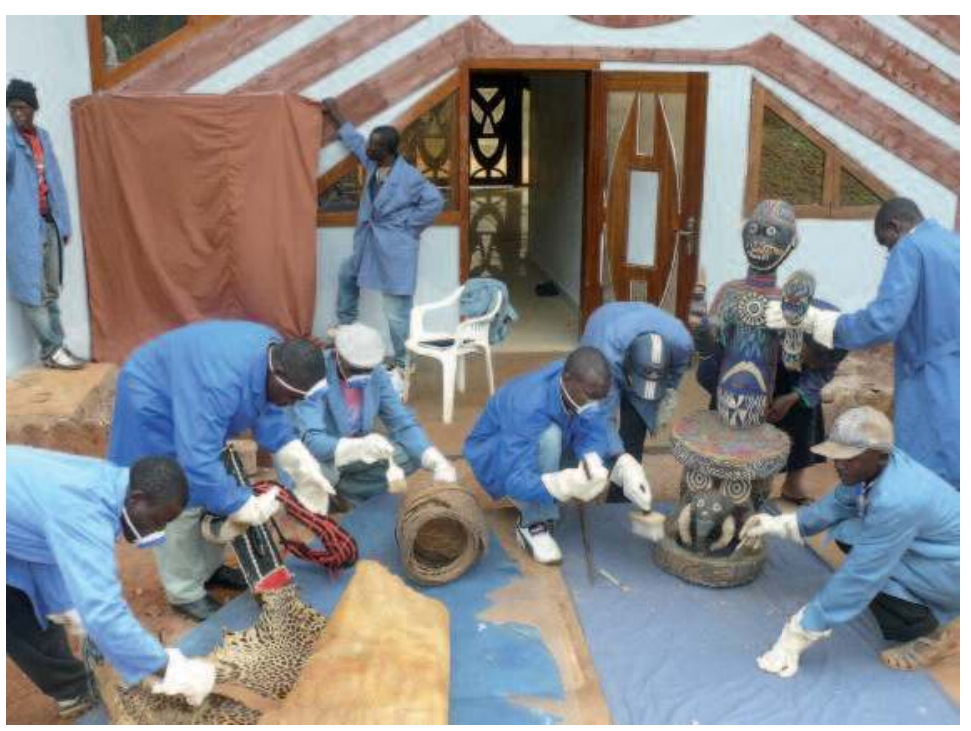

Une séance de traitement des objets par les étudiants de l'université de Dschang avant installation dans l'exposition du musée des Civilisations 


\section{Des difficultés aux premiers résultats très encourageants}

Un grand nombre de partenaires ont cru dans le projet et ont apporté leur contribution financière et technique ${ }^{(4)}$. Mais pour en arriver à cet état de réalisation, il a bien fallu surmonter un certain nombre d'épreuves tant au Cameroun qu'en France. Initialement nous avions du mal à être pris au sérieux par certains interlocuteurs du Nord. Le projet était trop ambitieux, pour un pays en voie de développement où la culture n'était pas prioritaire. Le projet ne relevait ni de la solidarité internationale, ni de l'humanitaire et pas complètement de la culture selon certains points de vue. De plus, nous défendions l'idée d'un projet de développement avec un des objectifs qui était de générer des ressources pour les populations locales à travers la valorisation du patrimoine et le développement touristique. Intégrer la dimension économique dans un projet de développement, porté par la société civile dont la diaspora camerounaise réunie au sein d'une association, était inconcevable pour certaines personnes du Nord.

Ce statut associatif nous a aussi valu de ne pas être considérés comme des professionnels, notamment des musées, car ne relevant pas de la fonction publique entre autres. Nous n'avions rien d'académique et en plus nous venions bousculer un fonctionnement et une certaine approche de la coopération internationale! Il faut aussi souligner que le PRDC piloté par la diaspora a bousculé des habitudes classiques des coopérations bilatérales et décentralisées pourtant préconisées dans de nombreux programmes de coopération mais peu mis en pratique.

Au Cameroun, le temps de la sensibilisation, de l'explication et de la discussion du concept du PRDC avec les chefs, leur communauté et la diaspora a été très long, mais n'a pas rencontré de difficultés particulières. Une fois compris, les chefs ont très vite adhéré au projet, le PRDC constituant une des réponses aux nombreux problèmes rencontrés par l'institution de la chefferie. La complexité de la mise en œuvre du projet a davantage résidé dans le contexte sociopolitique ne facilitant pas les choses.

Outre le manque de moyens financiers, nous avons aussi eu à faire face aux différences de temporalités : la question du temps du projet n'est pas la même au Sud qu'au Nord. Nous œuvrons bien entendu pour l'autonomie des acteurs et leur appropriation des activités, mais la difficulté réside davantage dans la méthodologie et l'organisation du travail. Le rôle des chefs aussi évolue, ils se retrouvent à la tête d'une

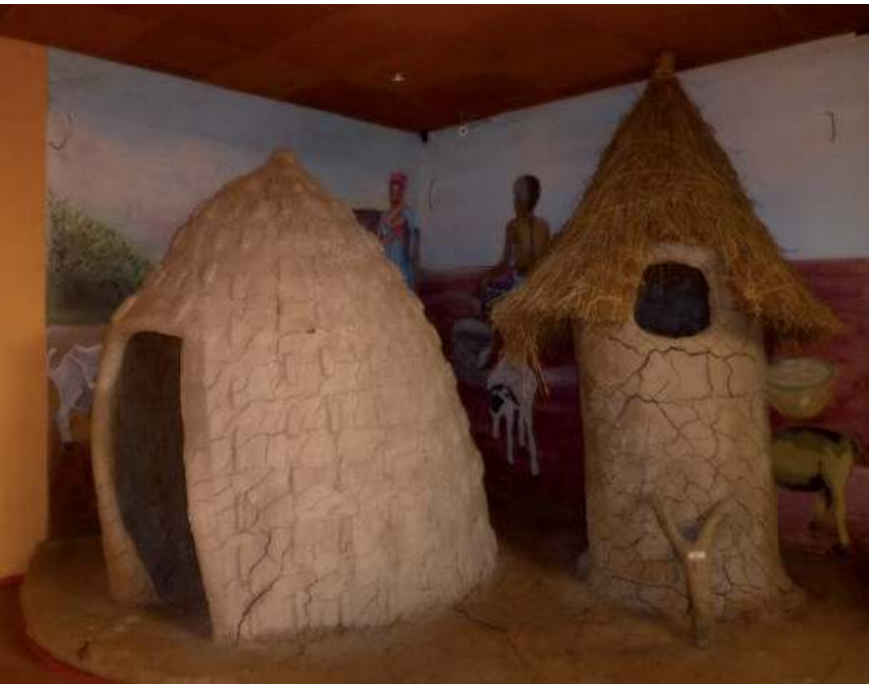

La reconstitution d'une case obus et d'un grenier mafa du Nord Cameroun au musée des Civilisations

(C) PRDC

institution muséale avec du personnel formé qui ne peut être gérée comme la chefferie. Les questionnements portent aujourd'hui sur la fonction et la place du chef dans son musée sans compter les difficultés liées au fonctionnement quotidien des cases patrimoniales.

Mais les résultats des premières activités sont très encourageants. En termes de fréquentation du musée des Civilisations, les statistiques de la première année sont très positives. Lors du week-end d'ouverture, organisé en parallèle d'une grande cérémonie traditionnelle à la chefferie Foto voisine du musée, 2200 visiteurs ont été enregistrés. L'entrée était gratuite. De fin novembre 2010 à novembre 2011, le musée a comptabilisé 8520 visiteurs, dont 93,50\% de visites payantes et $95 \%$ de visiteurs nationaux. Sur la totalité des visiteurs, 5899 jeunes (élèves, étudiants, touristes) ont visité le musée dont $96 \%$ dans le cadre d'une activité de médiation culturelle. Par ailleurs, une politique tarifaire très détaillée en fonction des différents publics cibles est pratiquée. L'objectif est de permettre au plus grand nombre de pouvoir visiter le musée.

Concernant l'objectif de sensibiliser les populations à leur histoire et identité culturelle, le pari semble également gagné au vu des témoignages inscrits dans le livre d'or. Beaucoup parlent de fierté nationale. Les visiteurs ne se sentent plus orphelins culturellement, ils sont impressionnés par l'ampleur des propos et exhortent à poursuivre l'initiative afin de réconcilier les générations. Le PRDC prouve ainsi que la protection du patrimoine n'est pas un frein à la modernisation. 
Ce succès manifeste attire de plus en plus l'intérêt des pouvoirs publics camerounais. Depuis quelques mois en effet, les hauts responsables de l'État (ministre du Tourisme, de la Jeunesse, de l'Enseignement supérieur et des Sports, gouverneur de la région, préfet du département, recteurs des universités du Cameroun...) effectuent des visites au sein du PRDC.

\section{Et la suite ?}

Le PRDC tire son originalité de la diversité des acteurs qui le composent, de son système de fonctionnement et surtout de la grande implication des communautés locales ; un succès qui réussit à émerger dans un contexte politique pourtant très complexe. Force est de croire que ce nouveau né dans le paysage patrimonial et touristique mondial, est le nouveau souffle dont a besoin le Cameroun pour émerger dans son secteur culturel et touristique. Il devient sur le plan national et même international un chantier source de propositions pour le développement culturel des pays africains en général.

La phase 2 de « La Route des Chefferies » qui couvrira la période 2012-2015 prévoit :

- la finalisation du Jardin des Civilisations, la poursuite de l'inventaire du patrimoine immatériel et matériel, le développement de la conservation préventive, la réalisation de nouveaux musées communautaires et de cases d'hôtes, des éditions sur le patrimoine ;

- la création de l'Agence de développement touristique de "La Route des Chefferies", avec comme vocation principale d'être un levier pour la promotion et le développement touristique et économique du territoire. Elle structurera le secteur en accompagnant les projets d'intérêt touristique et en mettant en œuvre une démarche qualité sur le label « La Route des Chefferies »;

- la mise en place du programme africain Infopatrimoine, prévoyant entre autres la numérisation du patrimoine, déjà partagé dans son concept par le Conseil Africain des Musées (AFRICOM), les professionnels du Bénin, du Mali, du Burkina-Faso, du Cameroun ;

- l'inscription du programme sur la liste indicative du patrimoine mondial de l'UNESCO.
(2) Somé R. et Weiss, G. La collection ethnographique de l'université de Strasbourg, la Lettre de l'OCIM, n¹34, mars-avril 2011, pp. 5-13.

(3) Djache Nzefa, S. Les chefferies Bamiléké dans l'enfer du modernisme... Une chefferie de demain... Couëron-France, 1994.

(4) Les partenaires du PRDC entre 2006 et 2011 sont : les villes de Nantes et de Dschang bénéficiant du contrat de plan État français Conseil régional des Pays de la Loire ; de l’Union européenne ; de l'École du Patrimoine Africain du Bénin ; du ministère du Tourisme du Cameroun; des mécènes et donateurs français et camerounais réunis au sein de l'Association des Amis du musée des Civilisations ; et prochainement par le Programme de Compétitivité des Filières de Croissance dans le cadre du prêt accordé par la Banque mondiale au Gouvernement camerounais.

\section{Bibliographie}

Djache Nzefa S. Les chefferies Bamiléké dans l'enfer du modernisme... Une chefferie de demain... Couëron-France, 1994.

Fagg, W. Sculptures africaines. Les univers artistiques des tribus d'Afrique noire. Paris : Fernand Hazan éditeur, 1965.

Felgine, O. Marché de l'art au bonheur des collectionneurs, Jeune Afrique Économie, 1991, n 147 .

Marchal, H. et Perrois, L. Les rois sculpteurs : art et pouvoir dans le grassland camerounais : legs Pierre Harter. Musée national des arts africains et océaniens, Réunion des musées nationaux, 1993.

Meauze, P. L'art nègre. Paris : Hachette, 1967.

Mefe, T. Cameroun : la culture sacrifiée, Africultures, ${ }^{\circ} 60$, juillet-septembre 2004, L'Harmattan.

Notué, J.-P. Batcham, Sculptures du Cameroun. Marseille : Musée de Marseille/Réunion des Musées Nationaux, 1993.

Réinventer les musées, Africultures, n70, juin 2007, L’Harmattan.

Taboué Nouayé, A.-F. Les artistes de Bandjoun, in Notué, J.-P. et Triaca, B. Bandjoun trésors royaux au Cameroun. Milan : 5 Continents Éditions, 2005, pp. 99-121.

Taffin, D. Du musée colonial au musée des cultures du monde, actes du colloque organisé par le musée national des Arts d'Afrique et d'Océanie et le Centre Georges-Pompidou, 3-6 juin 1998, Maisonneuve \& Larose, 2000 .

\section{Notes}

(1) Mefe, T. Cameroun : la culture sacrifiée, Africultures, $n^{\circ} 60$, juilletseptembre 2004, p. 15 